\title{
Situación de derechos de los niños, niñas y adolescentes trabajadores callejeros nocturnos en Medellín
}

\section{The Status of the Rights of Child and Adolescent Night Street Workers in Medellin}

\author{
John Fernando Ramírez Villegas \\ Yesenia Andrea Rojas Durango \\ Flor Ángela Tobón Marulanda ${ }^{1}$
}

\section{Resumen}

En este artículo se expone de manera general la problemática que enfrentan los niños, niñas y adolescentes — cuya sigla reconoceremos como NNA — trabajadores callejeros nocturnos de Medellín (Colombia). A partir de una investigación cualitativa con enfoque fenomenológico hermenéutico se logra observar e interpretar sus vivencias a la vez que se identifican mejor sus necesidades. La edad de los NNA a los que se les aplicó una entrevista y una guía de observación oscilaba entre los 9 y 14 años. El procesamiento de la información obtenida se realizó empleando el análisis de contenido, generando así algunas categorías desde los mismos datos. Dentro de los resultados se encontró que ellos tienen sus propias concepciones sobre la noche, el dinero, ser pobre, sus derechos, la libertad, la seguridad y la verdad, todas permeadas por una sociedad que ha sido indiferente a las necesidades de esta población, asimismo, este artículo genera una reflexión sobre el trabajo infantil en Colombia y reitera la necesidad de atender esta problemática social.

\section{Palabras claves}

Trabajo infantil, derechos de los niños, vulnerabilidad.

\section{Abstract}

This article presents, in general terms, the problems faced by child and adolescent night street workers in Medellin, Colombia - whose acronym we recognise as NNA -. From a qualitative study with a phenomenological hermeneutic approach we were able to observe and interpret their experiences while simultaneously identifying their needs. The ages of the children and adolescents who were interviewed and participated in an observation guide ranged from 9 to 14 years. The processing of the information obtained was performed using content analysis thus generating some categories from the same data. Among the results, it was found that they have their own ideas about the night, money, being poor, their rights, freedom, security and truth, all of which were permeated by a society that had been indifferent to the needs of this population. Likewise, this article also generates a reflection of child labour in Colombia and reiterates the need to address this social problem.

Keywords

Child labour, children's rights, vulnerability.

Artículo recibido el 14 de enero de 2014 y aprobado el 20 de agosto de 2014

1 Universidad de Antioquia, Medellín, Colombia. Correo electrónico: yeseniaudea@gmail.com 


\section{Introducción}

La infancia tiene sus propias maneras de ver, pensar y sentir; nada hay más insensato que pretender sustituirlas por las nuestras.

Rousseau

La Comisión Interamericana de Derechos Humanos $(\mathrm{CIDH})$ afirma que la problemática que vive la niñez colombiana frente a sus derechos no se debe a la falta de una legislación pertinente, al contrario, Colombia presenta un completo y sólido marco jurídico de los derechos de la niñez tanto a nivel nacional como internacional. La problemática radica específicamente en que todas las normas no se aplican a la situación real de la mayoría de nuestros NNA.

En consonancia con lo anterior, algunos estudios importantes de organizaciones no gubernamentales sobre la protección de los derechos de los NNA colombianos, los mismos que generaron la denuncia de la representante Ángela Robledo en el año 2011 sobre las presuntas irregularidades del Instituto Colombiano de Bienestar Familiar (ICBF) en la Comisión Séptima de la Cámara de Representantes (Robledo A., 1 de noviembre, 2011, comunicado de prensa), han llegado a la conclusión de que en nuestro país no existe un sistema estructurado y organizado de Bienestar Familiar y los programas de las entidades del sector oficial no son completamente eficaces.

La niñez colombiana sufre las severidades de la injusticia social debido a la falta de las condiciones mínimas para la protección de sus derechos fundamentales: alimentación, salud, vivienda, educación y la recreación, entre otros. Para la Comisión Interamericana de los Derechos Humanos (CIDH):

Lo más preocupante de la situación de la niñez colombiana es que el mismo derecho a la vida y a la integridad personal está siendo frecuentemente violado, pues cada vez son más los niños y las niñas víctimas de violación a estos derechos como consecuencia del conflicto armado interno o por la intolerancia social en la mal llamada "limpieza social". (Comisión Colombiana de Juristas, 1999, p. 347)
La Organización Internacional del Trabajo (OIT) considera que el trabajo infantil es una violación de los derechos humanos fundamentales en cuanto que vulnera el desarrollo físico, moral y psicológico de los NNA, y a nivel social perpetua su pobreza por generaciones. No obstante, es pertinente enunciar que aún no existe una definición jurídica uniforme sobre trabajo infantil que sea universalmente aplicable, ya que las normas internacionales del trabajo prevén excepciones y contienen cláusulas de flexibilidad para su aplicación, por lo tanto continúa abierta la discusión sobre si el trabajo infantil trasgrede o no los derechos de los niños.

El Departamento Administrativo Nacional de Estadística (DANE) incluye dentro de los niños en situación de trabajo infantil a todas las personas de 5 a 17 años de edad que, durante un período de referencia determinado, participan en una o más de las actividades siguientes: las peores formas de trabajo infantil (PFTI), el empleo por debajo de la edad mínima (15 años), y los servicios domésticos peligrosos no remunerados (DANE, 2012).

Según el Departamento Administrativo Nacional de Estadística (DANE) en la Encuesta Nacional de Trabajo Infantil, realizada en el año 2012, se encontró que de los $11332000 \mathrm{NNA}$, cerca de 1160000 trabajan y sus edades oscilan entre los 5 y 17 años. El 90 \% de los niños ejerce las PFTI, es decir, aquellas que ponen en peligro la salud y la vida de los menores: prostitución, pornografía, mendicidad, rebusque, reciclaje y actividades ilícitas.

Basado en cifras del 2009 el diagnóstico situacional de infancia y adolescencia de la Alcaldía de Medellín reporta que en la ciudad y su área metropolitana hay 682364 NNA trabajadores (Alcaldía de Medellín, 2012). De acuerdo con el censo de la Secretaría de Bienestar Social de la Alcaldía de Medellín, realizado en el mismo año, en las calles de Medellín existen aproximadamente 1080 NNA, de los cuales 927 no encuentran otra alternativa más que trabajar en la calle para contribuir con el sustento familiar y 153 son habitantes de la calle. ${ }^{2}$ Es

2 Se entiende por habitante de la calle a la persona de cualquier edad, que generalmente, ha roto en forma definitiva los vínculos con su familia y hace de la calle su espacio permanente de vida. 
importante señalar que el censo no especifica cuántos NNA trabajan en la noche (Alcaldía de MedellínSecretaría de Bienestar Social, 2009).

Aunque el gobierno colombiano en su Plan Nacional de Desarrollo 2010-2014 incluye dentro de los lineamientos estratégicos para generar un entorno de igualdad de oportunidades la implementación y el fortalecimiento de la estrategia de prevención y erradicación del trabajo infantil, según estadísticas de la Personería de Medellín en el año 2012, la situación lejos de mejorar, empeoró. Preocupa el incremento de la vulneración de los derechos de los NNA, ya que es insuficiente el presupuesto inicialmente previsto para las instituciones y esto, al final, se refleja en el detrimento en la calidad de la atención para el restablecimiento de sus derechos y por ende en una revictimización de los NNA (Personería de Medellín, 2012).

\section{¿Por qué hay tantos niños y niñas que se ven obligados a trabajar en la noche?}

Para Zuluaga (citado por la Organización de los Estados Americanos, 1999) los NNA que habitan las calles ${ }^{3}$ son víctimas de la violación y vulneración de sus derechos, debido a cuatro aspectos fundamentales:

- Socio-culturales: Las diferencias de clases sociales y la pobreza han generado una cultura de empleo informal en la cual están involucrados los NNA quienes luchan por sobrevivir y ayudar a sus familias.

- Violencia: Fenómenos como el maltrato físico, psicológico y verbal hacen que los NNA escapen de sus hogares y las comunidades en las que habitan.

- Familiares: El alto nivel de disfuncionalidad de las familias promueve la expulsión de los NNA a la calle, máxime si los padres consideran que estos no contribuyen económicamente.

3 Se entiende por habitante en la calle a la persona de cualquier edad, que hace de la calle el escenario propio para su supervivencia y la de su familia.
- Educacionales: Las escuelas de los barrios marginales no tienen en cuenta las demandas e intereses de la población infantil. Son excluyentes, intolerantes e incomprensivas con la realidad que asiste a los NNA y a sus familias.

El problema de los NNA que habitan las calles va más allá de las causas mediatas e inmediatas, ya que no son simplemente situaciones patológicas aisladas, sino que reflejan todo un desequilibrio económico y una descomposición social que tiende a incrementar la crisis que vive el país, convirtiéndose en una bomba de tiempo que requiere de decisiones efectivas que garanticen la protección de sus derechos.

Igualmente, Zuluaga (citado por la Organización de los Estados Americanos, 1999) clasifica a los NNA que habitan las calles en cinco categorías:

- Trabajadores: Son los NNA que se dedican a actividades de la economía informal, es decir, realizan ventas ambulantes de productos como golosinas, cigarrillos, chicles, entre otros. Estos conforman el mayor grupo poblacional de los NNA en la calle.

- Infractores: Son los NNA que se dedican al robo, atraco a mano armada y a la venta de psicoactivos, entre otras actividades ilícitas. Son altamente agresivos.

- Limosneros: Son los NNA dedicados a pedir dinero, generalmente lo hacen presionados por sus padres como medio de subsistencia.

- Explotados sexualmente: Son los NNA que adoptan como forma de vida y subsistencia la venta de su cuerpo.

- Gamines: Son los habitantes de la calle. NNA que viven en las calles de las ciudades, debajo de puentes y salen casi siempre en las noches, ya que las entidades de seguridad y los comerciantes de los lugares donde se camuflan les prohíben deambular durante el día. Se caracterizan por su ropaje harapiento y el consumo de drogas psicoactivas como el sacol (un tipo de pegante industrial), marihuana y bazuco, entre otras. 
El censo de la Secretaría de Bienestar Social de la Alcaldía de Medellín del año 2009 señala que los habitantes de la calle tienen como actividad central las PFTI y el trabajo sexual, mientras que los habitantes en la calle se orientan esencialmente a las ventas y los servicios ambulantes. En promedio el número de horas que ambos NNA dedican a las actividades anteriormente mencionadas supera las 8 horas diarias.

\section{¿Quién protege y previene la vulneración de los derechos de los NNA?}

La Convención sobre los Derechos del Niño (citada por la Organización de los Estados Americanos, 1999) señala que la familia es la institución responsable de garantizar la calidad de vida de los NNA, no obstante el Estado colombiano también juega un papel esencial en la protección de sus derechos ya que este "debe adoptar medidas apropiadas para ayudar a los padres a dar efectividad al derecho y, en caso necesario, proporcionar asistencia y programas de apoyo, particularmente con respecto a la nutrición, educación, vestuario y a la vivienda" (Comisión Colombiana de Juristas, 1999, p. 354).

Por lo tanto, si las condiciones económicas de las familias son precarias, el Estado está obligado a intervenir y a proveer a los NNA los medios para atender las necesidades básicas, en aras de evitar el pleno desarrollo de las cinco categorías laborales explicadas anteriormente.

Dentro de los programas del Estado y el gasto público debe ser prioritaria la atención a la niñez para garantizar y asegurar un nivel de vida adecuado, ya que por su condición de menores deben gozar de una protección especial que les garantice un pleno desarrollo integral. Se deben:

Adoptar las medidas necesarias para proteger al niño contra toda forma de perjuicio o abuso físico o mental, descuido o trato negligente [...] mientras el niño se encuentre bajo la custodia de los padres, de un representante legal o de cualquier otra persona que lo tenga a su cargo.(Comisión Colombiana de Juristas, 1999, p. 355)
La Comisión Interamericana de los Derechos Humanos (CIDH) afirma que si y solo sí el Estado colombiano cumple con la aplicación de la normatividad existente, podrá detener el desplazamiento de los NNA hacia el mundo de la calle, donde el nivel de vida es aún más precario, haciendo más obvia la necesidad de intervención y asistencia por parte del mismo.

\section{¿Quiénes son los niños, niñas y adolescentes trabajadores de la noche?}

Los NNA objeto de esta investigación trabajan los fines de semana entre las 6 p. m. y las 6 a. m. ejerciendo una de las Peores Formas de Trabajo Infantil (PFTI) según la clasificación de la Organización Internacional del Trabajo (OIT). La exposición en la noche a situaciones de alto riesgo, la violencia verbal y física, la escasa protección a las adversidades climáticas y físicas como el frío, la lluvia, el hambre, la sed, el cansancio y el sueño además de las extensas jornadas en la búsqueda de su sustento son condiciones que hacen que esta labor sea inaceptable y hostil para la condición humana y frágil de un niño.

En una ciudad que despierta de noche son latentes otros riesgos que no son tan evidentes en el trabajo infantil diurno, los NNA son víctimas potenciales del abuso sexual y del reclutamiento de grupos armados (combos y bandas criminales) que son un flagelo para la ciudad de Medellín.

La venta de dulces, rosas y sustancias psicoactivas son su principal fuente de ingreso. Sin embargo, estos son medios que una buena cantidad de los NNA utilizan como estrategia para atraer la atención de los adultos y prestarles servicios sexuales, generando cadenas de explotación sexual comercial infantil y adolescente.

Los NNA trabajadores de la noche huyen de los funcionarios del Instituto Colombiano de Bienestar Familiar (ICBF) y de la policía porque según ellos estos les vulneran el derecho al trabajo. La atención a tales casos consiste en una detención momentánea mientras se hace un llamado a los padres para que cumplan con la protección de sus hijos. 


\section{Metodología}

El acopio de la información se hizo a través de una guía de observación y una entrevista en profundidad aplicada a 15 NNA trabajadores nocturnos de la calle en la ciudad de Medellín, en sectores como Avenida la Playa en el Centro, la Avenida 70, el Parque Lleras en El Poblado y San Diego.

Por constituirse en un estudio cualitativo con enfoque fenomenológico hermenéutico, este es, ante todo, el relato de una vivencia reflexionada del mundo del trabajo infantil nocturno, y es también un espejo que refleja sus miedos, dilemas, razones y esperanzas.

Participan del estudio 10 niños y 5 niñas, 11 de los cuales tienen más de 12 años y 4 están entre los 11 y los 9 años. Ninguno ha roto sus lazos con las familias de origen o es habitante de la calle, y todos alternan el trabajo con el estudio. Son egocéntricos, tímidos y aún se refleja en sus actitudes la inocencia infantil.

Para el análisis se busca superar la presentación del dato, que es frecuente en estudios relacionados con esta temática. Se emplean entonces, para interpretar sus vivencias, la fenomenología y la hermenéutica las cuales se refieren a dos tradiciones filosóficas europeas cuyas nociones y aspectos metodológicos están siendo aplicados en la investigación cualitativa (Ayala, 2008). Las características más relevantes del contenido de los diálogos presentes en la entrevista son transformadas en unidades de análisis que luego son ubicadas dentro de siete categorías. La guía de observación permitió centrar la atención de los investigadores y hacer el registro de la información.

\section{Resultados}

Los menores trabajadores se dedican a mercadear el sueño de la noche, en los semáforos, bares y restaurantes de la ciudad de Medellín hacen malabares o venden dulces y rosas para poder realizar alguno de sus deseos u obligaciones:

Yo en el día le trabajo a mi mamá vendiendo Frunas y toda la plata es para la comida, y viernes y sábado le trabajo a mi mamita, vendiendo chicles, para que ella pague las deudas y ayude para los servicios y para la comida. (Entrevista personal, niña de 12 años, vendedora de chicles, sector la 70)

Ante la necesidad de contribuir con la economía familiar los NNA ocupan los roles de un adulto y día a día sin poder negarse, se responsabilizan de tareas que no les corresponden. Con frecuencia los NNA que trabajan en la noche se dedican a trabajos de alto riesgo y explotación, sin recibir ningún tipo de protección o regulación, algunos desempeñan labores que afectan su salud y ponen en peligro hasta su integridad física:

Unas veces los viejos morbosos le dicen a uno: “mami venga”, y yo “ipa' dónde?” [sic], entonces empiezan a mirar a uno, entonces a uno le toca insultarlo bien feo. (Entrevista personal, niña de 12 años, vendedora de confites. Sector La Playa)

Aunque en la ciudad de Medellín, como en otras tantas metrópolis, se ha desarrollado una imagen negativa de la noche, por enigmática y temida, para los NNA entrevistados tiene otras particularidades, para ellos este ambiente nocturno aunque peligroso también es agradable porque la gente se divierte y pueden hacer sus travesuras, a la vez es certidumbre porque es su medio de supervivencia:

Es oscura, es trabajo, es pa' [sic] hacer maldades, por ejemplo asustar a la gente, la noche es muy peligrosa porque hay más ventaja pa' [sic] los ladrones; está oscuro y las calles más despejadas y la gente que pasa es la víctima. (Entrevista personal, niño de 14 años, malabarista. Sector El Poblado)

Los NNA relatan que las travesuras consisten en asustar a la gente o robarle si se descuida, lo segundo no es un ejercicio habitual en las niñas. Sin embargo, los NNA tienen visiones oportunas de lo que la noche en realidad debería ser:

La noche es para estar en mi casa, me siento seguro en mi casa, me gusta mucho la noche, me gusta descansar relajada.(Entrevista personal, niña de 13 años, vendedora de confites y chicles. Sector La Playa)

La percepción del dinero en los NNA tiene otras características que la diferencian de la de los adultos, las niñas lo asocian con la sobrevivencia y los 
niños admiten que su dinero también puede ser gastado en mecato ${ }^{4}$ pero a ninguno le llama la atención emplearlo en viajes, bienes suntuarios, libros, drogas y licor; paradójicamente, algunos padres explotan los NNA utilizando irresponsablemente el dinero en sus adicciones.

En consonancia con lo anterior se indagó por la representación que los NNA tenían sobre qué es ser pobre sus respuestas son variadas y solo algunas parten de la autorreferencia, muchos de ellos no se proclaman pobres porque no son habitantes de la calle o viven en casas de tabla, barro o plástico.

Una persona pobre no es como nosotros, porque la verdad es que nosotros no somos pobres, una persona pobre es la que vive en la calle, como los indigentes, los gamines, los que tienen que coger basuras en las canecas para comer, esas son las personas pobres. (Entrevista personal, niña de 13 años, vendedora de confites y chicles. Sector La Playa)

Cuando se les pregunta a los NNA cuáles creen que son sus derechos, puede afirmarse que sus respuestas corresponden a un fuerte anhelo para la realización de sus derechos exigibles. Al margen de la importancia que le dan al derecho de la educación, prima el "derecho a ser niño", estar libres de responsabilidades, tener la garantía de desarrollarse y tener una familia que los valore y los respete.

Tenemos derecho a que los padres nos brinden amor y respeto, porque nosotros tenemos derecho a ser libres y no trabajar, a tener un poquito de libertad para jugar y divertirnos y no solamente a trabajar. (Entrevista personal, niña de 12 años, vendedora de chicles. Sector el Poblado)

Yo creo que tengo derecho a estudiar, porque los niños tenemos que dedicarnos al estudio. (Entrevista personal, niño de 13 años, vendedor de cristos. Sector la 70)

Muchos menores manifestaron el deseo de ser libres y tienen claridad de que el hecho de tener que trabajar es una privación de su libertad, así las

4 Término coloquial colombiano usado para referirse a dulces, pasabocas y alimentos que se emplean para calmar el hambre en forma temporal. cosas ellos recrean su espacio y reinventan la calle diseñando estrategias para gozar y divertirse mientras perciben algunas monedas para asegurar el sustento familiar. Los NNA idean juegos, cantan al ritmo de los artistas de los videos que se proyectan en una gran pantalla para los clientes de los bares y restaurantes y bailan al compás de las melodías que resuenan en la noche con su particular cadencia.

La percepción de seguridad varía según el género, las niñas se sienten más inseguras que los niños y renuncian a la protección de ellos, su necesidad de seguridad la resuelven en espacios llenos de gente, como el Parque del Periodista en el Centro de Medellín, donde experimentan la protección de los otros habitantes de la noche de este lugar, terminan haciéndose amigas de los mismos y de los dueños de los negocios y sus empleados para salvaguardarse del acecho continuo de aquellas personas que las quieren abusar sexualmente.

Me esfuerzo y me hago la enojada y por ejemplo, digámoslo así: el tipo lo va a seguir a uno y uno se siente como con miedo, uno lo lleva a una parte donde haya muy poca gente o también que haya mucha gente, lo hace caer bien mal y lo hace cascar ${ }^{5}$ por ahí de los amigos de uno, los mariguaneros esos o las lesbianas. (Entrevista personal, niña de 13 años, vendedora de confites y chicles. Sector La Playa)

Otro mecanismo de defensa en las niñas es el lenguaje vulgar, utilizan palabras soeces y de doble sentido para verse semejantes a los niños en aras de exigir el mismo respeto que les tienen a sus hermanos y compañeros de trabajo o para sentirse superiores al romper las reglas lingüísticas, desarrollándose una jerga que a veces es solo entendida por este grupo poblacional.

El lenguaje de los niños es menos fluido que el de las niñas, ellos son más parcos en sus respuestas y su vocabulario es escaso, lo cual podría ser una consecuencia de la situación de privación sociocultural y afectiva que viven en su cotidianidad.

5 El término cascar se entiende como golpear. 
Algunos NNA paradójicamente develan en sus expresiones el miedo a los organismos de control como la policía, el ICBF y Espacio Público, ya que los consideran como su principal amenaza:

La policía nos hace correr porque nos entramos a esos negocios, a esas cantinas, si uno está por ahí jugando llaman a la patrulla y se los llevan para Bienestar o le pegan pata a uno, lo cascan, nos dicen que si nos vuelven a ver ahí nos llevan para otras partes, nos llevan para la cárcel. (Entrevista personal, niño de 12 años, vendedor de chicles. Sector la Playa)

Esas autoridades que tienen como función controlar la venta de estupefacientes, prevenir el robo, denunciar el trabajo infantil y capturar a los presuntos responsables de delitos, entre muchas otras funciones, terminan invisibilizando la pobreza, la inequidad y la realidad, escondiendo el deber ser de una sociedad equitativa, donde se revela el malestar que producen sus propias incapacidades. La calle es un espacio que esconde el trabajo infantil y/o adolescente nocturno, lo hace anónimo impidiendo que se consoliden sistemas de trabajo decente y diluyendo la posibilidad de generar mejores sistemas de protección social para los NNA.

Los menores en condición de trabajadores de la calle desarrollan habilidades y destrezas que les permiten sufrir con entereza las adversidades de la noche, sin dejarse doblegar fácilmente ante las circunstancias de la vida. Su capacidad resiliente para afrontar su inhumana realidad desafía los límites de su edad.

Ellos poseen una habilidad especial para la mentira y el secreto, la cual es usada comúnmente para su autoprotección en la noche. Su manera de asumir la verdad se corresponde con sus vivencias, los NNA construyen una moralidad ajustada a sus necesidades y van aprendiendo a discernir cuando, donde y a quienes contar sus secretos; además, se observa que ocultan información u objetos para evitar el abuso o generar compasión, por ejemplo: a las niñas que se les preguntaba: “ ¿Usted con quién está por ahí a estas horas?” Respondían: “Con mis hermanos mayores", los niños les mostraban a los clientes la caja donde cargaban las cajitas de chicles casi vacía para motivar la compra generada por la lástima que se produce en el transeúnte frente una realidad tan lamentable como esta.

\section{Discusión}

La niñez trabajadora en Colombia es víctima de la explotación laboral, la mitad de ellos no recibe salario y la otra mitad no alcanza a devengar medio salario mínimo legal vigente. Esta población se enfrenta a actividades de alto riesgo como la minería, la construcción y la recolección de basura, entre otras, de igual forma, a quehaceres ilícitos como el sicariato, la prostitución, el expendio de drogas y alucinógenos.

Dentro de las causas principales del trabajo infantil se encuentran la marginalidad en que vive la mayoría de esta población y la descomposición familiar que hace que los niños asuman desde edades muy tempranas obligaciones de tipo económico. A esto se suma la pobreza como factor sociocultural que se constituye en un problema de supervivencia para los menores trabajadores. Este último factor, asociado al hambre, determinan las precarias condiciones en las cuales se desenvuelven los NNA, privándose de oportunidades para educarse y vivir su infancia en un ambiente adecuado.

El menor debería estar con su familia compartiendo un espacio apropiado para su desarrollo y crecimiento afectivo, social y emocional, pero nuestra situación real exige que los NNA asuman funciones laborales que además de vulnerar sus derechos se caracterizan por jornadas de trabajo que exceden los límites permitidos (48 horas semanales) y salarios por debajo de lo establecido en la norma (Ley 50 de 1990). Además, en el trabajo infantil no se reconocen las prestaciones sociales mínimas a que tiene derecho cualquier trabajador colombiano.

Esta modalidad laboral transgrede dos obligaciones del Estado, erradicar las peores formas de trabajo infantil (PFTI) y garantizar el acceso y la permanencia de los NNA en el sistema educativo. Desde la Organización Internacional de Trabajo (OIT) se plantea como paliativo la fijación y aplicación de una edad mínima en la que los NNA pueden 
ser admitidos en algunos tipos de trabajos ligeros, la cual no debería ser inferior a la edad en que se completa la enseñanza escolar obligatoria.

En este orden de ideas, el Fondo Internacional de Emergencia de las Naciones Unidas para la Infancia (Unicef) afirma que en tanto se mejore cuantitativa y cualitativamente el sistema escolar y se ofrezcan apoyos a las familias más desprotegidas, los NNA no tendrán que ejercer actividades laborales, ilegales y absurdas.

Según el análisis de la situación de los NNA trabajadores en Colombia, la Comisión Interamericana de los Derechos Humanos (CIDH) plantea algunas recomendaciones en aras de mejorar las condiciones de vida de la población infantil:

- Otorgar la debida importancia a los temas referentes a los derechos de los niños y niñas, teniendo en cuenta que el problema no es solo la inversión económica sino más bien el reconocimiento y compromiso social y político frente al problema.

- Adoptar medidas claras que difundan los derechos de la niñez, especialmente entre los niños, los padres, los defensores de niños, los maestros, los jueces, los policías, los militares, los grupos de profesionales que trabajan con niños y niñas.

- Crear un sistema que coordine la aplicación de los programas relacionados con la infancia y que se consolide al Sistema Nacional de Bienestar Familiar, con miras a lograr una buena coordinación entre las instituciones que se ocupan de los derechos del niño. Además, se hace necesario evaluar sistemáticamente progresos a nivel cuantitativo y cualitativo realizados.

- Reconocer y brindar importancia a las instituciones no gubernamentales que velan por el cumplimiento de los derechos de la niñez, e igualmente sean incentivados en la medida de lo posible con la participación en el diseño, elaboración y ejecución de las políticas estatales en pro de los niños y las niñas.
- Invertir los recursos económicos suficientes en los servicios prioritarios que garanticen la salud y la educación de la infancia.

- Generar programas de alfabetización, cobertura que involucre a los niños y niñas en el sistema educativo escolar.

- Profundizar en los programas que protegen a los niños y niñas del conflicto armado interno.

- Poner en práctica las reformas necesarias frente a la jurisdicción de familia.

A menudo el trabajo infantil es minimizado por el adulto ya que se tiene una percepción equivocada del mismo, este a veces se ve como una colaboración o ayuda familiar o como una simple tarea que el NNA realiza a tiempo parcial, visión que va legitimando esta acción convirtiendo lo ilegal en legal.

Los NNA que trabajan en la calle en la noche se configuran como actores dentro de este marco, no obstante sus actividades se inscriben en un contexto peligroso, donde ellos construyen nuevas significaciones que estimulan una mirada hacia ellos mismos y hacia los otros. Sus percepciones sobre la noche, el dinero, ser pobre, sus derechos, la libertad, la seguridad y la verdad, como lo demuestra este trabajo, adquieren otros sentidos que les permiten sobrellevar su infortunada vivencia.

Son escasas las discusiones acerca del trabajo infantil callejero nocturno, frecuentemente se cae en su condena automática, sin embargo, es necesario profundizar en su análisis para que se visibilice y desde toda perspectiva se busquen alternativas a fin de erradicar esta problemática social que se está perpetuando en el tiempo a través de la indolencia social y la incompetencia estatal.

\section{Conclusiones}

Los derechos de los NNA son vulnerados cada vez más, otorgándoles menos garantías. La solución a esta problemática no está en interpelar leyes más justas, sino en plantear estrategias necesarias para que se cumplan las ya existentes. 
Los NNA trabajadores callejeros nocturnos deben ser reconocidos como seres humanos portadores de demandas sociales y sujetos de derecho, que no solo asuman el papel de beneficiarios, sino que se les garantice la protección desde todas las esferas y no terminen finiquitando los deseos de los adultos, sino que puedan ellos mismos realizar sus propios sueños.

Concernirá al Estado, a la familia y a la escuela ayudar y proteger el desarrollo integral del NNA, de manera que adquieran progresivamente autonomía en el ejercicio de sus derechos y gocen de estos que a bien han sido establecidos por la Constitución Política Colombiana.

Finalmente, se comparte la conclusión razonada por la Oficina Regional de la Unicef (citada por la OEA, 1999) en el sentido de que el trabajo infantil "es tal vez el más extendido fenómeno que atenta contra el bienestar y el normal desarrollo de la infancia”. En el trabajo infantil callejero nocturno y sus actores, caracterizados por ser o parecer ser sujetos fuertes, astutos, sagaces, valientes, maduros, habituados y acostumbrados al siniestro mundo de la noche con sus implicaciones como el miedo, la violencia, la injusticia, la opresión y el desorden, se refleja todo el desequilibrio, la descomposición social y las condiciones de marginalidad de una sociedad en crisis de valores como la nuestra, que no es justa con ellos.

\section{Referencias}

Alcaldía de Medellín (2012). Diagnostico situacional de la infancia y adolescencia en el municipio de Medellín. Recuperado de:

http://www.medellin.gov.co/irj/go/km/docs/wpccontent/sites/subportal\%20del\%20ciudadano/bienestar\%20social/secciones/informes/documentos/2012/ diagn\%c3\%b3stico\%20de\%20infancia\%20y\%20adolescencia\%20nuevo\%20formato.pdf

Alcaldía de Medellín-Secretaría de Bienestar Social (2009). Informe final censo de habitantes de calle $y$ en calle de la ciudad de Medellín y sus corregimientos. Recuperado de: http://aprendeenlinea.udea.edu.co/ revistas/index.php/ceo/article/viewfile/7073/6486
Ayala, R. (2008). La metodología fenomenológico-hermenéutica de M. Van Manen en el campo de la investigación educativa. Posibilidades y primeras experiencias. Revista de Investigación Educativa, 26(2), 409-430. Recuperado de: http://www.usergioarboleda.edu.co/lumen/biblioteca/Raquel\%20Ayala/ Metodolog\%C3\%ADa\%20fenomenol\%C3\%B3gicohermen\%C3\%A9utica\%20de\%20Manen\%20en\%20 Investigaci\%C3\%B3n\%20Educativa.pdf

Comisión Colombiana de Juristas (2009). Derechos humanos en Colombia. 3er Informe de la Comisión Interamericana de Derechos Humanos. Recuperado de:

http://www.coljuristas.org/documentos/libros_e_informes/ddhh_en_colombia_3er_informe_de_la_ CIDH.pdf

Departamento Administrativo Nacional de Estadística (2012). Informe trabajo infantil octubre-diciembre 2012. Recuperado de: https://www.DANE.gov.co/ index.php/es/mercado-laboral/trabajo-infantil/91sociales/mercado-laboral/5488-informacion-historica-trabajo-infantil

Henao, G., Gómez, S., Ocampo, L., y Ramírez, J., (2008). Por el derecho al sueño (Tesis de Maestría). cinde, Medellín, Colombia.

Organización de Estados Americanos (1999). Tercer informe sobre la situación de derechos humanos en Colombia. Recuperado de: http://www.CIDH.org/ countryrep/colom99sp/capitulo-13.htm

Personería de Medellín (2012). Informe sobre la situación de los derechos humanos en la ciudad de Medellín 2012. Recuperado de:http://www.personeriamedellin.gov.co/documentos/INFORME_D1.pdf

Robledo, A. (1 de noviembre, 2011).Directora convirtió al ICBF en un "monstruo" contratista. Recuperado de:

http://www.angelarobledo.com/Comunicaciones/ ComunicadosdePrensa/tabid/430/mid/1141/articleId/2008/ctl/ReadAngelaRobledo/language/ en-US/Default.aspx 